\title{
Weak lensing of CMB temperature and polarization patterns: implications for large-scale structure
}

\section{Michael Kesden ${ }^{*}$ CITA}

E-mail: kesden@cita.utoronto.ca

The primordial CMB is weakly lensed by large-scale structure (LSS) between us and the lastscattering surface, changing both the temperature and polarization power spectra and inducing higher-order non-Gaussian correlations. These non-Gaussian correlations can be exploited to reconstruct the weak-lensing deflection field, with a statistical detection possible by Planck at high significance. The reconstucted lensing field can be used to study the LSS itself, and is essential for removing secondary $B$-mode polarization to constrain primordial tensor modes produced during inflation.

Ischia, Italy

${ }^{*}$ Speaker. 


\section{Introduction}

A wealth of current and future experiments including the upcoming Planck mission will provide us with an all-sky map of both the temperature and polarizations patterns in the CMB. The primordial power spectrum derived from existing data has done much to confirm the $\Lambda \mathrm{CDM}$ concordance model of the Universe, and has allowed us to estimate the cosmological parameters of this model with unprecedented precision. The low-noise, high-resolution maps that will become available in the near future will allow us to study not just the primordial CMB generated at the lastscattering surface but also secondary anisotropies produced in the intermediate and high-redshift Universe. Though these secondary anisotropies can be viewed as "foregrounds" from the perspective of estimating parameters from the primordial $\mathrm{CMB}$, considered in their own right they can teach us much about cosmology and the formation of large-scale structure (LSS). The subject of this talk will be the secondary anisotropies induced by weak lensing, the deflection of primordial CMB photons by the LSS between us and the last-scattering surface. Measurements of weak lensing's effect on the CMB are important for a variey of reasons. Weak lensing's dependence on LSS implies that it is yet another constraint the primordial power spectrum $P(k)$ and thus a valuable consistency check on estimates of $\sigma_{8}, n_{s}$, and other cosmological parameters. Unlike galaxy lensing, weak lensing of the CMB is sensitive to the LSS all the way back to the last-scattering surface, providing a rare window on the largest-scale (lowest $k$ ) modes. To the extent that these modes are uncorrelated with the primordial CMB they are invaluable in helping to beat down the cosmic variance that plagues all attempts to compare experiment with theory at these scales. Finally, reconstruction of the lensing field out to the last-scattering surface is potentially essential to recovering primordial $B$-mode polarization, often described as a "smoking gun" of gravitational waves generated during inflation.

We will attempt to provide some qualitative insight into the effect of weak lensing on CMB temperature and polarization in Section 2, and how this lensing differs from the lensing of background galaxies very familiar to the astronomy community. Section 3 will then discuss several of the recently developed semi-analytic techniques for exploiting lensing-induced non-Gaussianity in the CMB to reconstruct the lensing-deflection field. We will begin with an analysis of the temperature and polarization-based quadratic estimators valid to linear order in the lensing field $\phi$, which should allow at least a statistical detection of the lensing power spectrum for an experiment of the noise and sensitivity of Planck. We then show that to higher order in $\phi$ these estimators are biased, and that this bias will be relevant for next-generation experiments such as CMBPOL. Maximum likelihood estimators, though computationally more cumbersome, reveal the limitations of quadratic estimators and will be necessary to extract optimal limits from these next-generation experiments. The application of the these reconstruction techniques to constraining primordial tensor perturbations and their relevance to near-future experiments is considered in Section 4, where it is seen that reconstruction can push upper bounds on the tensor-to-scalar ration $r$ down from $r \lesssim 10^{-2}$ to as low as $10^{-5}$ assuming the very difficult task of foreground removal can be achieved. Some concluding remarks are given in Section 5. 


\section{Weak Lensing}

A nice pedagogical treatment of the effect of weak lensing on the CMB is given in [1]. The trajectories of CMB photons are governed by the geodesic equation,

$$
\frac{d^{2} x^{i}}{d \lambda^{2}}=-\Gamma_{\alpha \beta}^{i} \frac{d x^{\alpha}}{d \lambda} \frac{d x^{\beta}}{d \lambda}
$$

where the Christoffel symbols $\Gamma_{\alpha \beta}^{i}$ can be derived from the perturbed metric

$$
d s^{2}=-(1+2 \Psi) d t^{2}+a^{2} \delta_{i j}(1+2 \Phi) d x^{i} d x^{j} .
$$

These perturbations lead to a remapping of the observed temperature and polarization fields, so that observations in a direction $\theta^{i}$ correspond to a point in a direction $\theta_{S}^{i}$ at the last-scattering surface given by the deflection field

$$
d^{i} \equiv \theta_{S}^{i}-\theta^{i}=2 \int_{0}^{\chi} d \chi^{\prime} \Phi, i\left(\vec{x}\left(\chi^{\prime}\right)\right)\left(1-\frac{\chi^{\prime}}{\chi}\right) .
$$

which to excellent approximation can be expressed as the gradient of a lensing potential $d^{i}=$ $\nabla^{i} \phi$. Integrating Eq. (2.3) out to the last-scattering surface results in typical deflections of several arcminutes in magnitude. The simple fact that lensing manifests itself as a remapping has important implications for how we might hope to detect its effects in the observed CMB. The deflection field at a given point depends on the LSS integrated along the line-of-sight, not on whether the primordial CMB is hot or cold in that particular direction or how the polarization vector is oriented. As a result, lensing cannot making the mean CMB hotter or colder, or induce any changes in the onepoint probability distribution function (PDF). This symmetry further implies that lensing cannot generate a three-point or other odd correlation function on its own, though correlations between the lensing field and other secondary anisotropies like the Sunyaev-Zeldovich (SZ) effect that trace the LSS can lead to such correlations. One of the most generic predictions of inflation, confirmed to high accuracy by WMAP and other experiments, is that the primordial CMB should be Gaussian, implying that its statistcs are fully determined by its power spectrum and that the connected parts of all higher-order correlation functions such as the trispectrum must vanish. By remapping the primordial CMB lensing not only shifts power from large to small scales, but more importantly for the purpose of lensing reconstruction generates a non-zero trispectrum that in the next section will be shown to be intimately connected to efforts to determine the lensing-potential power spectrum.

Weak lensing affects polarization patterns in the CMB in ways very similar to its effect on the temperature, but with a very significant new complication. In the absence of tensor modes generated during inflation, primordial CMB polarization consists entirely of $E$-modes in which the gradient of the polarization field is either parallel or perpendicular to the direction of the polarization itself. This follows from the fact that the only direction specified by scalar perturbations is their gradient, and therefore symmetry compels both the polarization and its gradient to align in that direction as well [1]. By contrast, tensor perturbations or gravitational waves (GWs) have an intrinsic polarization themselves, and thus the two different directions specified by the GWs' gradient and polarization allow the directions of the resulting CMB polarization and gradient to become decoupled. Both $E$ and $B$ polarization modes are necessary to describe this more general 
polarization pattern. In a similar way to GW polarization, gradients in the lensing potential field supply supply a second direction and thus necessitate the use of $B$-modes to characterize the resulting lensed CMB polarization field. The $B$-mode polarization induced by lensing must be removed as accurately as possible to set the tightest limits on primordial tensor modes, a subject we will return to in Section 4.

Additional insight into the effects of weak lensing on the CMB can be gained by comparison with the weak lensing of a field of background galaxies. One major difference is that background galaxies exist over a broad redshift distribution, while the CMB source plane is fixed at the lastscattering surface. While this redshift distribution does offer the tempting possibility of lensing tomography, uncertainty in the peculiar velocities of galaxies poses an additional complication for lensing reconstruction using galaxies as sources. Galactic lensing reconstruction also does not have access to the largest-scale modes probed by the CMB, as the redshift distribution of galaxies peters out long before it reaches the last-scattering surface at $\mathrm{z} \simeq 1100$. The other major difference is that the fundamental limits of galactic lensing reconstruction are set by the finite number density of galaxies, uncertainty in their ellipticities, and any possible intrinsic ellipticity alignments, while the limits on CMB lensing reconstruction derive from the uncertainty in which Gaussian realization of the primordial temperature and polarization the Universe has chosen to adopt. The observed CMB temperature map alone simply doesn't contain enough information to uniquely constrain both the primordial CMB temperature map and the lensing-potential field. However, under the assumption of neglible primordial $B$-mode polarization, the observed $E$ and $B$-modes could be used in principle to determine both the primordial $E$-modes and the lensing field. Reconstruction is still not possible for $l \gtrsim 2000$ where Silk damping begins to cut off primordial power and there is no information to remap.

\section{Lensing Reconstruction}

Our discussion of the effects of lensing in the previous section gives us some insight into early efforts to reconstruct the lensing field from the observed CMB. Metcalf and Silk [2] noted that weak lensing shifted large-scale power in the CMB temperature field down to scales at which primordial power was strongly suppressed by Silk damping. They therefore proposed that measurements of the CMB power spectrum at these small scales would be proportional to the power spectrum of the lensing potential itself. Bernardeau [3] suggested an approach similar to that of galactic lensing reconstruction, where tangentially sheared galaxies such as those famously seen in the Hubble Deep Field are interpreted as indications of a projected mass concentration. The same phenomenon should occur for patches in the CMB temperature map, whose elongations on average should be isotropic but could exhibit an alignment when lensed by a large-scale mode of the deflection field. Further progress was made when Zaldarriaga and Seljak [4] discovered that to linear order in the lensing field, Fourier modes of the map of the CMB temperature at each point squared were estimators for the same Fourier mode of the deflection field. Hu [5] generalized this approach to all quadratic temperature estimators, and showed that one particular statistic corresponding to the product of the temperature and its gradient was optimal to linear order. $\mathrm{Hu}$ and Okamoto then constructed quadratic estimators from polarization observables as well [6], and derived full-sky versions of these estimators using spherical harmonic coefficients in place of Fourier modes [7]. 
As these estimators should have great practical importance for Planck and future experiments, let's look at them a bit more closely.

\subsection{Quadratic Estimators}

Quadratic estimators of the lensing potential $\phi$ are based on the fact that two Fourier modes $\mathbf{l} \neq-\mathbf{I}^{\prime}$ of the CMB observables $X, X^{\prime}=\Theta, E, B$ are uncorrelated in the Gaussian primordial CMB, but to linear order in $\phi$ are correlated exclusively by the mode $\mathbf{L}=\mathbf{l}+\mathbf{I}^{\prime}$,

$$
\left\langle X(\mathbf{l}) X^{\prime}\left(\mathbf{I}^{\prime}\right)\right\rangle_{\mathrm{CMB}}=f_{X X^{\prime}}\left(\mathbf{l}, \mathbf{I}^{\prime}\right) \phi(\mathbf{L}),
$$

where \langle\rangle$_{\mathrm{CMB}}$ represents an ensemble average over the primordial CMB, and the function $f_{X X^{\prime}}\left(\mathbf{I}, \mathbf{I}^{\prime}\right)$ depends on instrumental noise and the primordial CMB power spectra. The amplitude of $f_{X X^{\prime}}\left(\mathbf{I}, \mathbf{I}^{\prime}\right)$ and thus the strength of the correlation varies with the scale and configuration in a known way, as does the signal-to-noise with which the modes $X(\mathbf{I}), X^{\prime}\left(\mathbf{I}^{\prime}\right)$ can be observed. Hu and Okamoto [6] derived optimal quadratic estimators

$$
\mathbf{d}_{X X^{\prime}}(\mathbf{L}) \equiv \frac{i \mathbf{L} A_{X X^{\prime}}(L)}{L^{2}} \int \frac{d^{2} \mathbf{l}_{1}}{(2 \pi)^{2}} \frac{1}{2}\left[X\left(\mathbf{l}_{1}\right) X^{\prime}\left(\mathbf{l}_{2}\right)+X^{\prime}\left(\mathbf{l}_{1}\right) X\left(\mathbf{l}_{2}\right)\right] F_{X X^{\prime}}\left(\mathbf{l}_{1}, \mathbf{l}_{2}\right)
$$

for a mode $\mathbf{L}$ of the deflection field by combining all pairs of modes $\mathbf{l}_{1}, \mathbf{l}_{2}$ for which $\mathbf{l}_{1}+\mathbf{l}_{2}=\mathbf{L}$ with weights given by the Wiener filter $F_{X X^{\prime}}\left(\mathbf{l}_{1}, \mathbf{l}_{2}\right)$ and normalization $A_{X X^{\prime}}(L)$. Six correlated estimators $\mathbf{d}_{X X^{\prime}}(\mathbf{L})$ can be constructed from the three observables $\Theta, E, B$, which themselves can be combined into a single minimum-variance $(\mathrm{mv})$ estimator. The performance of the individual and mv estimators is shown in Fig. 1 for both Planck and a future reference experiment with similar resolution but better sensitivity $\left(\Delta_{T}=\Delta_{P} / \sqrt{2}=1 \mu \mathrm{K}\right.$-arcmin [6]. This figure reveals that Planck does not have low enough sensitivity to reconstruct individual modes of the deflection field, but that a future experiment like CMBPOL could conceivably reconstruct modes out to $L$ of several hundred. Since Planck can't reconstruct individual modes $\mathbf{d}(\mathbf{L})$, it must settle for a statistical detection of the deflection-field power spectrum binned over a range of $L$.

\subsection{Power Spectrum Estimation}

Naively, one might hope that an estimator for the deflection-field power spectrum could be created by combining estimators for the individual modes in an appropriate manner,

$$
D_{L} \equiv \frac{(2 \pi)^{2}}{A L^{2}} \frac{1}{2 \pi L \Delta L} \int_{a_{L}} \frac{d^{2} \mathbf{l}}{(2 \pi)^{2}} \mathbf{d}_{X X^{\prime}}(\mathbf{l}) \cdot \mathbf{d}_{X X^{\prime}}(-\mathbf{l})
$$

where the individual modes are binned in an annulus $a_{L}$ of radius $L$ and width $\Delta L$. This hope is dashed by the fact that terms beyond linear order in $\phi$ yield a systematic contribution to $D_{L}$, even averaged over different realizations of the primordial CMB and LSS [8]. These terms lead to a subdominant contribution to the noise with which individual modes can be reconstructed as shown in Fig. 2, but constitute a systematic bias for estimations of the power spectrum if not properly subtracted. They can be interpreted as the noise associated with reconstructing a mode $\mathbf{d}(\mathbf{L})$ due to uncertainty in the other lensing modes. Since this noise depends on the deflection-field power spectrum itself, it must be subtracted iteratively though these iterations will rapidly converge 


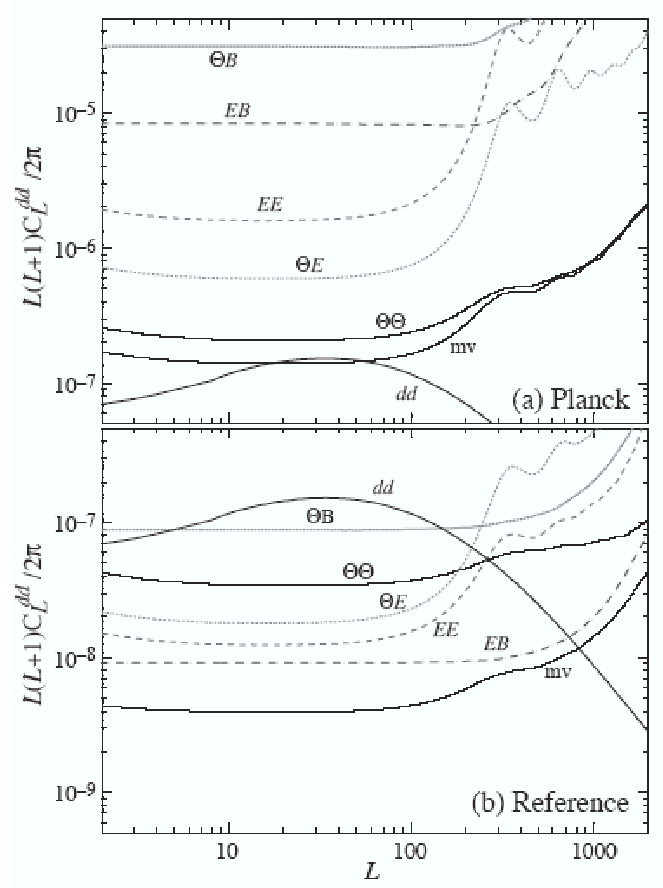

Figure 1: Variances of quadratic estimators of the deflection field $\mathbf{d}(\mathbf{L})$ for Planck and a future reference experiment. The curves $d d$ represent the expected deflection-field power spectrum, while the other curves correspond to the noise power spectra of the appropriately labeled estimators and their minimum varinace (mv) combination. Figure borrowed from [6].

as the effect only occurs at the $10 \%$ level even for the reference experiment. It is important to note that while the primary noise depicted in Fig. 2 is dominated by instrumental noise and thus decreases dramatically for the reference experiment, the second-order term is largely independent of instrumental noise and thus will become increasingly significant for future experiments. Despite this second-order noise, Planck should be able to determine the deflection-field power spectrum out to $L$ of several hundred with temperature and polarization [6]. To assess the ultimate limit of future experiments like CMBPOL we need to go beyond quadratic estimators to more recently developed maximum-likelihood techniques.

\subsection{Maximum Likelihood Estimators}

Hirata and Seljak [9] brought a new level of computational rigor to lensing reconstruction by devising a new estimator that exploited the full effects of the lensing remapping

$$
\Lambda[\phi] \Theta(\mathbf{n})=\Theta(\mathbf{n}+\nabla \phi(\mathbf{n}))
$$

rather than just its linear approximation. This remapping when combined with the assumed Gaussian statistics of the primordial CMB fully determines the covariance of the observed temperature $\hat{\Theta}(\mathbf{n})$ treated as a column vector,

$$
C^{\hat{\Theta} \hat{\Theta}}[\phi]=\Lambda[\phi] C^{\Theta \Theta} \Lambda[\phi]^{T}+C^{n} .
$$




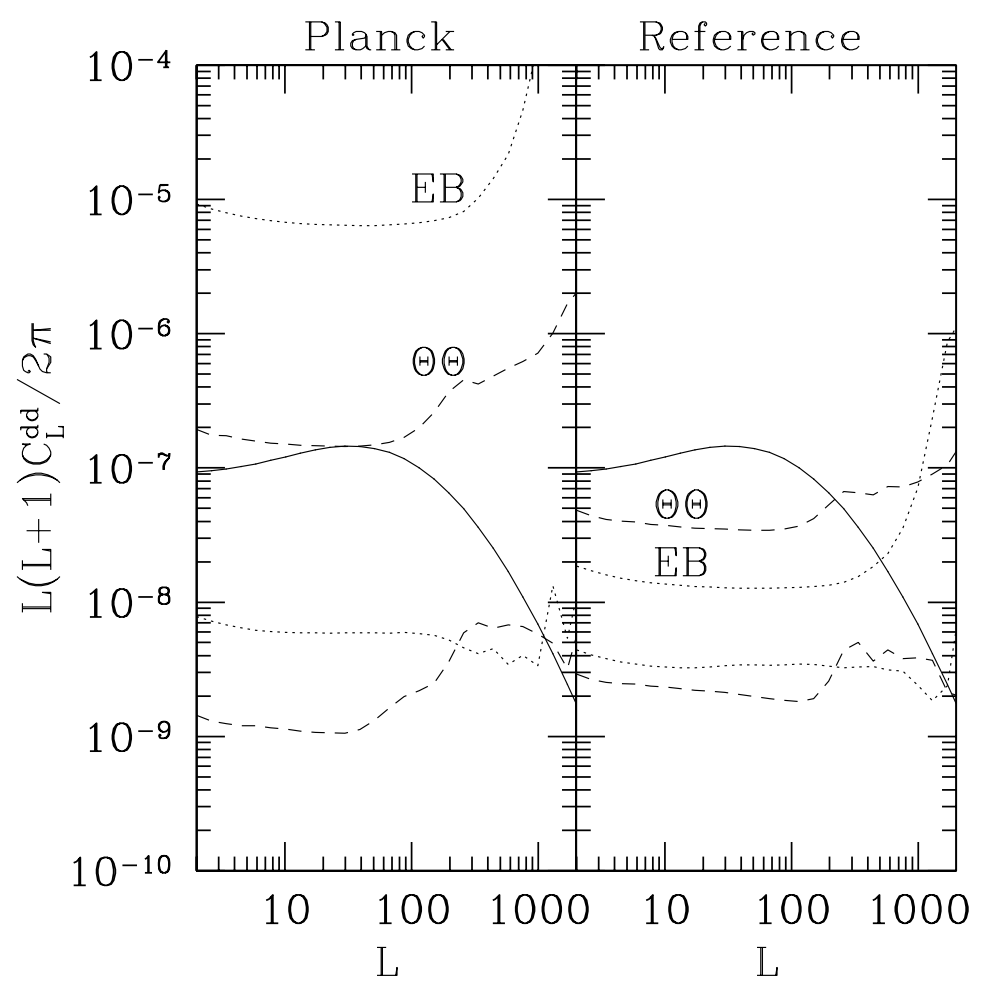

Figure 2: Primary and second-order contributions to the noise of the $\Theta \Theta$ and $E B$ deflection-field estimators for Planck and a future reference experiment. The solid curves are the power spectra $C_{L}^{d d}$. The upper and lower dashed curves are the primary and second-order noise power spectra for the temperature-based estimator, while the dotted curves are the corresponding noise variances for the $E B$ polarization estimator. Figure borrowed from [8].

This covariance is a functional of the realization of the lensing potential $\phi(\mathbf{n})$ from which the negative log likelihood $\mathscr{L}$ can be calculated. The maximum-likelihood estimator (MLE) $\hat{\phi}(\mathbf{n})$ is then the solution to the $N$ equations $\delta \mathscr{L} / \delta \phi=0$ for an $N$-pixel map. If the lensing potential $\phi(\mathbf{n})$ is itself assumed to be a realization of a Gaussian field with power spectrum $C^{\phi \phi}$, then the power spectrum must be determined iteratively as with the quadratic estimators described above. The result of applying this new estimator is shown in Fig. 3, and is in quantitative agreement with what we would expect from our study of nonlinear effects in the preceding subsection. The maximumlikelihood estimator offers little improvement for Planck, where instrumental noise dominates that associated with the confusion of lensing modes. It can offer however roughly a $10 \%$ improvement for a future reference experiment with dramatically improved sensitivity.

Maximum-likelihood techniques can be applied to polarization-based estimators in a very similar fashion, but with an important new conclusion [10]. As discussed in Section 2, the absence of significant power in the primordial $B$-polarization field implies that there is enough information in principle in the observed $E$ and $B$ fields to reconstruct both the primordial $E$ field and the lensing realization $\phi$. The noise associated with nonlinear effects sets a limit to how well quadratic polarization estimators can reconstruct the lensing field, but polarization-based MLEs can circumvent 


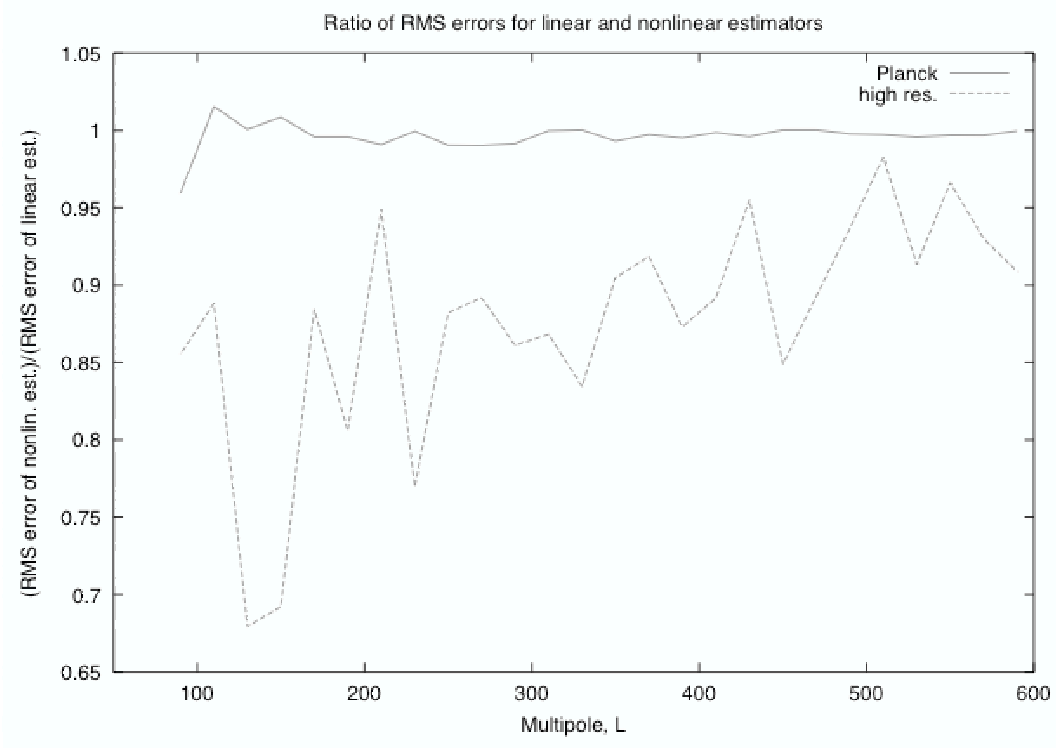

Figure 3: Ratio of the RMS error associated with the temperature maximum-likelihood and quadratic estimators for the lensing potetial $\phi$ as a function of $L$ for both Planck and a future reference experiment. Figure borrowed from [9].

this limit as shown in Fig. 4. The noise associated with the quadratic estimator saturates for experiments with instrumental noise lower than $1 \mu \mathrm{K}$-arcmin, but the performance of the MLE keeps improving until it has achieved an almost order-of-magnitude reduction in the reconstruction noise for Experiment F of Fig. 4 (0.25 $\mu \mathrm{K}$-arcmin, 2 arcmin resolution). This improvement has fundamental implications for an important application of lensing reconstruction, constraining primordial tensor modes.

\section{Constraining Tensor Modes}

As noted in Section 2, one of the most important theoretical developments in the study of CMB polarization was the discovery that primordial $B$-modes would be a "smoking-gun" signature of inflationary gravitational waves $[11,12]$. More recently it was found that lensing induced a fractional conversion of $E$-modes into $B$-modes,

$$
\tilde{B}(\mathbf{l})=-\int \frac{d^{2} \mathbf{l}_{1}}{(2 \pi)^{2}} E\left(\mathbf{l}_{1}\right) \sin 2\left(\varphi_{\mathbf{l}_{1}}-\varphi_{\mathbf{l}}\right) \phi\left(\mathbf{l}-\mathbf{l}_{1}\right)\left[\left(\mathbf{l}-\mathbf{l}_{1}\right) \cdot \mathbf{l}_{1}\right],
$$

contaminating the signal from primordial tensor perturbations for values of $r$ much below $10^{-2}$. Fortunately, lensing reconstruction can be used to estimate the lensing contribution to observed $B$-modes from Eq. (4.1), allowing these lensed modes to be removed from any primordial signal $[13,14]$. We have already seen that Planck will not be able to achieve lensing reconstruction on the mode-by-mode basis necessary for this application, but future experiments like CMBPOL should be able to do so. The limits to which these experiments can constrain $r$, assuming optimistically that foregrounds can be understood and controlled, will be set by lensing reconstruction using optimal 

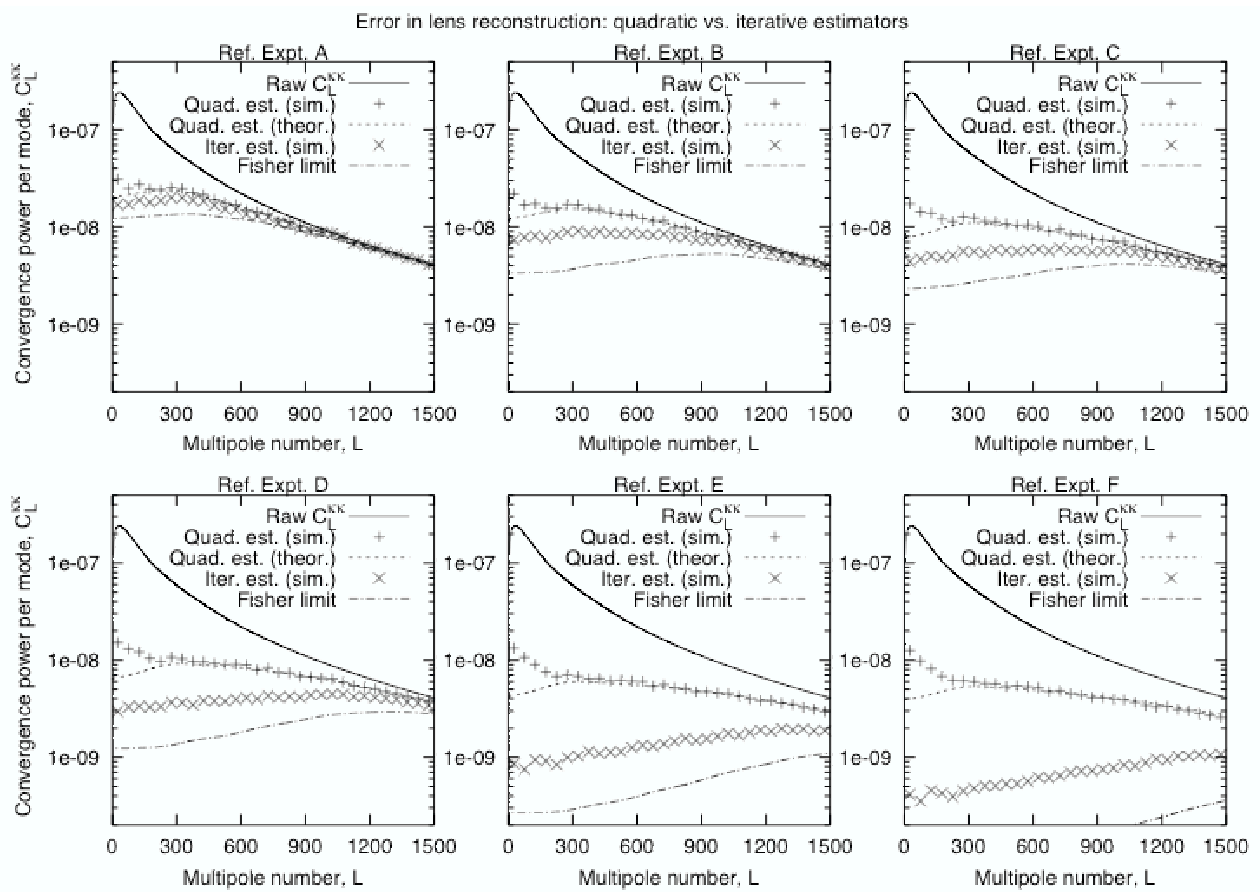

Figure 4: Error with which modes of the convergence field can be reconstructed by polarization-based estimators for future reference experiments. The solid curves show the signal convergence power spectrum, while the "+" and " $\times$ " data points show the quadratic and MLEs respectively. Noise decreases and resolution increases in the series from A to F. Figure borrowed from [10].

estimators like the MLEs of the previous section [15]. The effectiveness of these estimators is illustrated in Fig. 5, where we see the primordial $B$-mode polarization power spectra for two values of $r$ and the upper bounds one might hope to set with lensing reconstruction by quadratic estimators and MLEs. The optimistic $r=10^{-6}$ curve seeks to take advantage of the high first-year WMAP optical depth that has since come down. Nonetheless, an ultimate limit for $r$ of order $10^{-5}$ seems feasible in the absence of foregrounds.

\section{Conclusions}

This talk has sought to provide some insight into the effects of weak lensing on the CMB, and how these effect might be exploited to reconstruct both the primordial CMB and the lensing field itself. Lensing remaps the $\mathrm{CMB}$ on scales of several arcminutes, inducing distinctive non-Gaussian correlations and fractionally converting $E$-mode polarization to $B$-modes. Quadratic estimators adopt a linear approximation for the effects of lensing, then make use of the simple form of linear effects in Fourier or harmonic space to optimally weight all pairs of temperature and polarization observables coupled by a given lensing mode. MLEs retain the full nonlinear effects of lensing at the cost of having to estimate the power spectrum through an iterative Monte Carlo procedure. Planck should be able to achieve a statistical detection of the lensing power spectrum using quadratic estimators, with little improvement from using the more intensive MLEs. Future exper- 


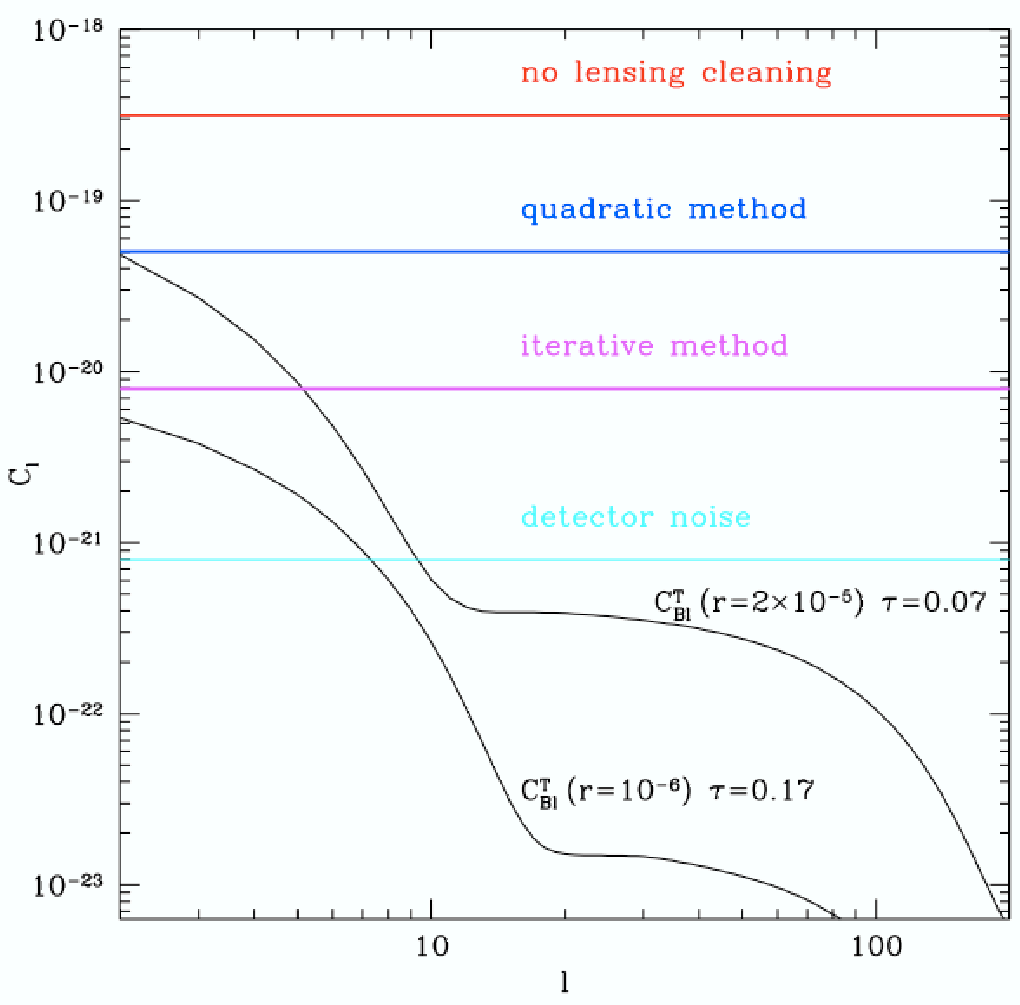

Figure 5: Residual $B$-mode polarization power spectra with no lensing subtraction (red), subtraction with quadratic estimators (blue), MLEs (magenta), and perfect subtraction (cyan). We see that a reference experiment along the lines Experiment $\mathrm{F}$ could conceivably detect primordial $B$-modes for $r$ as low as $10^{-5}$. Figure borrowed from [15].

iments attempting to set fundamental limits will achieve substantially better results using MLEs, and could conceivably detect primordial $B$-modes for $r$ as low as $10^{-5}$.

\section{References}

[1] Dodelson, S., Modern Cosmology, New York: Academic Press, 2003.

[2] R. B. Metcalf and J. Silk, Astrophys. J. 489, 1 (1997).

[3] F. Bernardeau, Astron. \& Astron. 338, 767 (1998).

[4] M. Zaldarriaga and U. Seljak, Phys. Rev. D 59, 123507 (1999).

[5] W. Hu, Phys. Rev. D 64, 083005 (2001).

[6] W. Hu and T. Okamoto, Astrophys. J. 574, 566 (2002).

[7] T. Okamoto and W. Hu, Phys. Rev. D 67, 083002 (2003).

[8] M. Kesden, A. Cooray, and M. Kamionkowski, Phys. Rev. D 67, 123507 (2003).

[9] C. M. Hirata and U. Seljak, Phys. Rev. D 67, 043001 (2003).

[10] C. M. Hirata and U. Seljak, Phys. Rev. D 68, 083002 (2003). 
[11] U. Seljak and M. Zaldarriaga, Phys. Rev. Lett. 78, 2054 (1997).

[12] M. Kamionkowski, A. Kosowsky, and A. Stebbins, Phys. Rev. Lett. 78, 2058 (1997).

[13] L. Knox and Y. Song, Phys. Rev. Lett. 89, 11303 (2002).

[14] M. Kesden, A. Cooray, and M. Kamionkowski, Phys. Rev. Lett. 89, 11304 (2002).

[15] C. M. Hirata and U. Seljak, Phys. Rev. D 69, 043005 (2004). 